\title{
Probabilistic Investigation of Sensitivities of Advanced Test-Analysis Model Correlation Methods
}

\author{
Elizabeth J. Bergman \\ Matthew S. Allen \\ Daniel C. Kammer \\ Department of Engineering Physics \\ University of Wisconsin \\ Madison, WI 53706 \\ Randall L. Mayes \\ ${ }^{1}$ Sandia National Laboratories \\ PO Box 5800 \\ Albuquerque, NM 87185
}

\begin{abstract}
Correlation of test and analysis mode shapes using reduced Test Analysis Models (TAMs) has become an industry standard method used to validate finite element models. Some organizations have even mandated specific metrics regarding the allowable deviations in the orthogonality of mode shapes, using the TAM mass matrix, that a model must meet to be considered valid. As a result, significant effort has been directed towards developing robust TAMs and in comparing the robustness of the various approaches. The static or Guyan reduction method has been touted as the most robust, although the evidence in the literature seems quite inconclusive. This work takes a new view on test analysis orthogonality, utilizing a probabilistic framework to accurately quantify the sensitivity of the Static, Modal and IRS reduction methods to errors in a set of mode shapes for a general satellite FEA model. The results show that the orthogonality calculation is highly sensitive to errors, so that the Static and IRS TAM models have a very small probability of passing the orthogonality criteria, when the true mode shapes are used, contaminated by only a small level of noise. This study also shows that the performance of the Modal TAM depends heavily on the sensor set chosen; the sensitivity of the modal TAM was observed to increase fourfold when a Static TAM sensor set was used rather than one that was optimized specifically for the Modal TAM. The results are also evaluated in light of one theory that relates sensitivity to the natural frequencies of the system constrained at the sensor locations, revealing that the theory fails for the Modal TAM yet may perhaps hold for the IRS TAM. While a conclusive ranking of the TAM methods cannot be achieved using a single model, this work presents the tools, based on a probabilistic framework, that can be used to correctly ascertain the sensitivity of test analysis models and to evaluate to model validation methodology.
\end{abstract}

\section{INTRODUCTION}

Finite element models have been used successfully for many years to predict the response of structures to their environmental loads. The finite element approach has been the workhorse for the aerospace structural dynamics community for decades. In order to ensure that critical predictions are accurate, the finite element model (FEM) must be experimentally validated. The experiment is usually a vibration test where the natural frequencies and normal modes of the structure are extracted from measured acceleration response. The state-of-the-practice for determining the accuracy of FEM predictions is based on comparing test and FEM modal parameters. This process has been called test/analysis correlation. Frequencies are compared directly, while the corresponding mode shapes are compared using various metrics. The metrics most often employed are the orthogonality and cross-orthogonality of the modes. Due to the mismatch in the number of FEM degrees of freedom and sensors used in a modal test, direct computation of modal orthogonality is not possible. A reduced representation of the

\footnotetext{
${ }^{1}$ Sandia is a multi-program laboratory operated by Sandia Corporation, a Lockheed Martin Company, for the U.S. Department of Energy under Contract DE-AC04-94AL85000.
} 
FEM, called a test-analysis model (TAM) must be generated. The orthogonality of the modes can then be computed with respect to the reduced analytical mass matrix, or TAM mass matrix [1]. The use of these metrics, and the required values for test/analysis correlation, are dictated by agencies such as NASA [2] and the United States Air Force [1]. Depending on the agency, the requirements are different. For example, the Air Force requires test/analysis frequency errors less than or equal to $3.0 \%$, cross-generalized mass values greater than 0.95 , and coupling terms between modes of less than 0.10 in test-orthogonality and test-FEM cross-orthogonality.

Many model reduction techniques have been developed, but the one that has been used the longest, and perhaps trusted the most, is the static or Guyan reduction [3]. However, there are cases where it does not provide accurate representations of the FEM, such as solid rocket motor applications [4]. This provided incentive for the development of a series of new more accurate advanced TAM techniques, using Modal [5], Hybrid [6], IRS [7], and SEREP [8] reductions. The Modal, Hybrid, and SEREP TAMs exactly predict the target modes, while the IRS TAM improves on the static TAM by approximating the inertia terms that are neglected in the static reduction.

While it is accepted that these advanced reduction techniques more accurately predict the FEM modal parameters, and greatly improve orthogonality when the test mode shapes are equal to the FEM mode shapes, it is commonly believed that they are more sensitive to FEM/test errors than the standard Guyan reduction. Sensitivity produces larger off-diagonal terms in the orthogonality computations, and this sensitivity is thought to be a fault of the TAM rather than a meaningful indication of mismatch between the test results and the FEM. This is undesirable, not only from the analyst's point of view, but also from the test engineer's perspective, where success of the test is based on minimizing the size of these terms. A literature survey reveals a few works that suggest that the advanced TAMs are more sensitive to errors in the test data, many others that are inconclusive, and some that appear to be inconclusive yet seem to blindly promote the claim. Chung [9] used real modal test data with the sensor locations optimized for the static reduction. He compared the static, IRS, Modal, and Hybrid TAMs for three different systems. He concluded that overall, the static TAM performed the best, but the differences between the cross orthogonality terms for all of the methods were small for all three systems considered. Freed and Flanigan [10] investigated the same TAM representations for two different examples. The first used simulated test data based on a modified FEM representation of a simple spacecraft. Sensors were placed based on kinetic energy and mode shape observability. They found that the Hybrid and Modal TAM representations were the most sensitive to errors. The second example used real test data from the Titan dual payload. In this case, the Hybrid TAM was the most robust with respect to errors, while the static TAM was better than the IRS TAM. The Modal TAM was the most sensitive. Avitable, et. al. [11] compared static, IRS, MAC [12], SEREP, and Hybrid TAM approaches. They used both analytically simulated and real test data for a 2-bay truss. In the case of simulated experimental data, they found that the SEREP and Hybrid TAMs produced better orthogonality results than the static TAM for large sensor sets, and significantly better results for reduced sets. For real test data, SEREP, Hybrid, and static TAMs gave similar orthogonality results for large sensor sets, but the static TAM was significantly worse for small sensor sets.

There is some evidence suggesting that the Modal TAM may be overly sensitive under certain circumstances. There may be several causes for this and few, if any, have been thoroughly investigated in the literature. For example, the question of sensor locations is very important. Most studies comparing various TAM representations use the same sensor set for each TAM, usually chosen for the static TAM. However, each reduction procedure demands its own optimally placed sensor set. The modal TAM requires that the target modes partitioned to the sensor set are as linearly independent as possible. A method such as Effective Independence [13] can be used to select the proper set of sensors. The static reduction also requires that the target modes are independent, but in a mass weighted sense. The use of a non-optimal sensor set could be a source of the Modal TAM sensitivity. Another possible source of sensitivity in the Modal TAM may be due to the lack of residual compliance. While the Modal TAM exactly predicts the target modes used in its production, the prediction of residual modes is totally inaccurate. The Modal TAMs residual basis vectors may not be adequate to describe the difference between the FEM and test target modes. Kammer [6] addressed this problem by combining the Modal and static TAM representations to produce the Hybrid TAM.

Yet another source of sensitivity was proposed by Gordis [14]. He stated that sensitivity can be explained by the dynamic reduction equation. Dynamic reduction is ill-conditioned at or near any frequency that corresponds to a natural frequency of the structure with all the sensor degrees of freedom constrained. The IRS TAM approximates the exact dynamic reduction, so Gordis concluded that it should also be sensitive to test errors in the neighborhood of the constrained structure frequencies. He showed that this was the case for the IRS TAM 
both mathematically, and through numerical examples. He then concluded that any advanced TAM method that attempts to approximate the inertia forces that are missing from the static TAM would suffer from this sensitivity problem. This claim was not directly investigated for either the Modal or Hybrid TAMs, yet the claim has been applied to them and repeated in recent works [15].

This paper uses a simple numerical example to explore the question of TAM sensitivity, or lack of robustness, to test data errors. The approach is new in that the sensitivity is studied using a probabilistic framework. Previous works have employed a deterministic approach, computing the orthogonality of the test modes with respect to the TAM once, for a single set of test data. This work recognizes that the modal parameters extracted in a modal survey can be expected to vary if the test were repeated, so they can be modeled as random variables. The magnitude of the variability can be very significant, especially for the mode shapes and damping ratios. Uncertainty associated with the FEM parameters, test boundary conditions, etc... can also be included in this description. The effect of uncertainty is evaluated by corrupting a set of FEM target modes with uniform random errors and then performing a Monte Carlo simulation in which, at each iteration, the orthogonality of the noisy modes is computed with respect to the TAM being studied and statistics are computed for the maximum offdiagonal terms, average absolute off-diagonal terms, etc. Probability distributions for these quantities can then be estimated describing the sensitivity of each TAM to noise.

The most important result of this work is that it presents the tools needed to provide conclusive answers to questions regarding TAM sensitivity, whereas the previous works provided only a single sample of the complex random process. It is important to note that, like many previous papers, only a single structural system is considered here, so the question of which TAM is best cannot be conclusively answered. However, the framework presented here can be used to understand the issues involved with TAM sensitivity and test/FEM correlation, and to develop remedies. By utilizing a probabilistic approach, this work reveals that when sensitivity to errors is important, passing or failing the orthogonality criteria may be purely a matter of random chance. Furthermore, the probability of failing to meet test-orthogonality criteria may be high, even if the FEM is accurate and the errors in the test mode shapes are small.

Failing to recognize the role of uncertainty can cause endless headaches for both experimentalists and analysts. If the experimentalists find that their initial test modes do not satisfy test-orthogonality, they are likely to invest extra time and effort in the test, perhaps adjusting boundary conditions, modifying modal parameter identification, checking sensor orientations, etc... This adds to the cost of the test, but would add little value if the initial test modes failed test-orthogonality only because of the small errors that are always present in a test. Likewise, when analysts encounter a model that has failed to meet validation criteria due primarily to an overly sensitive TAM, they may:

- Reject an FE model that is accurate to within the resolution of the test measurements.

- Needlessly update a FEM model to recreate the random noise pattern observed in a particular test.

- Make endless perturbations to the TAM procedure, sensor set, or FEM model in an effort to pass the validation criteria, achieving little more than a random set of model perturbations that passes the orthogonality metric for a given set of noise contaminated mode shapes.

- Falsely conclude that an inadequate model is valid because it correlates well with a particular test.

This paper is organized as follows. Section 2 briefly reviews the theory underlying the TAM generation methods used here. Sections 3 and 4 describe the system of interest and the sensor placement schemes that were used. Section 5 presents the pre-test FEM-TAM correlation results, and Section 6 presents the mode shape error model and its effect on test-orthogonality. Conclusions are presented in Section 7.

\section{Theory}

Three types of TAMs are utilized in this study; the static or Guyan TAM [3], the Improved Reduced System (IRS) TAM [7], and the Modal TAM [5]. For each TAM the degrees of freedom that are retained are denoted as the "aset" and hence the corresponding matrices have a subscript of "a". The omitted degrees of freedom are denoted as the "o-set" and the corresponding matrices have a subscript of "o".

\subsection{Static (Guyan) TAM}

The Static TAM is the most common model reduction technique. It is simple to implement with the algorithm built in to many commercial finite element codes. The static model reduction can be derived beginning with the equations of motion for a structure partitioned into the a-set and o-set degrees of freedom. 


$$
\left[\begin{array}{ll}
M_{a a} & M_{a o} \\
M_{o a} & M_{o o}
\end{array}\right]\left\{\begin{array}{l}
\ddot{x}_{a} \\
\ddot{x}_{o}
\end{array}\right\}+\left[\begin{array}{cc}
K_{a a} & K_{a o} \\
K_{o a} & K_{o o}
\end{array}\right]\left\{\begin{array}{l}
x_{a} \\
x_{o}
\end{array}\right\}=0
$$

For the ith mode shape and frequency, the FEM eigenvalue problem can be written as

$$
-\omega_{i}^{2}\left[\begin{array}{ll}
M_{a a} & M_{a o} \\
M_{o a} & M_{o o}
\end{array}\right]\left\{\begin{array}{l}
\phi_{i a} \\
\phi_{i o}
\end{array}\right\}+\left[\begin{array}{ll}
K_{a a} & K_{a o} \\
K_{o a} & K_{o o}
\end{array}\right]\left\{\begin{array}{l}
\phi_{i a} \\
\phi_{i o}
\end{array}\right\}=0
$$

Extracting the lower partition gives

$$
\left\lfloor K_{o a}-\omega_{i}^{2} M_{o a} \mid\left\{\phi_{i a}\right\}+\left\lfloor K_{o o}-\omega_{i}^{2} M_{o o} \backslash\left\{\phi_{i o}\right\}=0\right.\right.
$$

One can reproduce the motion of the structure at the o-set degrees of freedom from the a-set motion by solving this equation for $\left\{\phi_{i o}\right\}$ resulting in the following

$$
\left\{\phi_{i o}\right\}=-\left[K_{o o}-\omega_{i}^{2} M_{o o}\right]^{-1}\left[K_{o a}-\omega_{i}^{2} M_{o a}\right]\left\{\phi_{i a}\right\}
$$

However, this solution for the o-set is dependent on the reduction frequency. The Static TAM neglects the inertia terms and hence removes the dependency of the o-set solution on frequency. The resulting Static transformation is thus given by

$$
\left[T_{S}\right]=\left[\begin{array}{c}
I \\
-K_{o o}^{-1} K_{o a}
\end{array}\right]
$$

The statically reduced mass and stiffness matrices are formed using

$$
\begin{aligned}
\tilde{M}_{S} & =\left[T_{S}\right]^{T}[M]\left[T_{S}\right] \\
\widetilde{K}_{S} & =\left[T_{S}\right]^{T}[K]\left[T_{S}\right]
\end{aligned}
$$

Each column of $\left[T_{S}\right]$ represents the elastic deformation of the structure under a unit displacement of the corresponding a-set dof with all others constrained. These shapes are also referred to as constraint modes.

\subsection{Improved Reduced System TAM}

The Improved Reduced System (IRS) TAM [7] is a model reduction method that aims to improve upon the Static TAM by approximating the neglected inertia terms. The frequency dependent terms in Eq. (4) are approximated using the statically reduced mass and stiffness matrices.

$$
\omega_{i}^{2}\left\{\phi_{i a}\right\} \approx \widetilde{M}_{S}^{-1} \widetilde{K}_{S}\left\{\phi_{i a}\right\}
$$

The IRS transformation matrix can be written as the Static transformation matrix plus a correction term, $\left[T_{i}\right]$.

$$
\left[T_{I R S}\right]=\left[T_{S}\right]+\left[T_{i}\right]
$$




$$
\left[T_{i}\right]=-\left[\begin{array}{cc}
0 & 0 \\
0 & -K_{o o}^{-1}
\end{array}\right]\left[\begin{array}{cc}
M_{a a} & M_{a o} \\
M_{o a} & M_{o o}
\end{array}\right]\left[\begin{array}{c}
I \\
-K_{o o}^{-1} K_{o a}
\end{array}\right] \widetilde{M}_{S}^{-1} \widetilde{K}_{S}
$$

The reduced mass matrix and stiffness matrices, $\tilde{M}_{I R S}$ and $\widetilde{K}_{I R S}$, are computed as in Eq. (6) and (7).

The IRS TAM is an approximation to the dynamic reduction given in Eq. (4). One can see that the dynamic reduction equation depends upon inverting the matrix

$$
\left[K_{o o}-\omega_{i}^{2} M_{o o}\right]
$$

which is ill-conditioned when $\omega_{i}^{2}$ is near any of the eigenvalues of the $K_{\circ o}, M_{\circ o}$ system. In other words, if constraining the system's a-set degrees of freedom produces a system whose eigenvalues are near the natural frequencies of the actual system, the dynamic reduction equation will be ill-conditioned and one might not be able to solve for the o-set motion from the a-set responses. Gordis [14] noted this fact and suggested that the IRS TAM might greatly magnify errors in the test mode shapes if this is the case because the IRS TAM approximates the dynamic reduction equation. He suggested that one can test for sensitivity by computing the eigenvalues of the system with the a-set DOF restrained and see if the resulting frequencies are close to the frequencies of the actual system.

\subsection{Modal TAM}

There are circumstances in which the Static TAM fails to accurately represent the dynamics of the full system because it neglects the mass associated with the o-set degrees of freedom. For instance, the solid rocket motor application [4] contained modes that were dominated by massive and soft propellant. The Static TAM neglected terms that were significant to the analysis and thus did not provide a good representation of the system. The modal TAM was developed to resolve this issue. The method uses the modal expansion equation for the target modes

$$
\{x\}=[\phi]\{q\}
$$

where $[\phi]$ represents the target modes, and $\{q\}$ represents the generalized modal coordinates. This equation can be partitioned into the a-set and o-set degrees of freedom.

$$
\left\{\begin{array}{c}
x_{a} \\
x_{o}
\end{array}\right\}=\left[\begin{array}{c}
\phi_{a} \\
\phi_{o}
\end{array}\right]\{q\}
$$

This produces two matrix equations.

$$
\begin{aligned}
& \left\{x_{a}\right\}=\left[\phi_{a}\right]\{q\} \\
& \left\{x_{o}\right\}=\left[\phi_{o}\right]\{q\}
\end{aligned}
$$

The first equation can be solved for the modal response vector $\mathrm{q}$. 


$$
\{q\}=\left[\phi_{a}^{T} \phi_{a}\right]^{-1}\left[\phi_{a}^{T}\right]\left\{x_{a}\right\}
$$

This requires that the matrix $\left[\phi_{a}^{T} \phi_{a}\right]^{-1}$ be invertible, so the number of a-set degrees of freedom must be greater than or equal to the number of target modes and the target FEM modes partitioned to the sensor locations must be linearly independent. When Eq. (16) is substituted into Eq. (15) the transformation matrix for the Modal TAM is given by

$$
\left[T_{M}\right]=\left[\begin{array}{c}
I \\
\phi_{o}\left(\phi_{a}^{T} \phi_{a}\right)^{-1} \phi_{a}^{T}
\end{array}\right]
$$

The reduced mass matrix $\tilde{M}_{M}$ is computed in the usual way. The primary advantage of the Modal reduction method is that it is exact for the modes used in the reduction process.

\section{Model Description}

The system of interest for this paper is the generic satellite shown in Fig. 1 The satellite is unconstrained and therefore has six rigid body modes. The finite element model is comprised of 1,191 nodes totaling 7,146 degrees of freedom. The target modes chosen for this study are the first eighteen elastic modes listed in Table 1. Fig. 2 displays elastic modes 1 and 2 . The core of the satellite contains five lumped masses that have relatively small displacements in each mode shape.

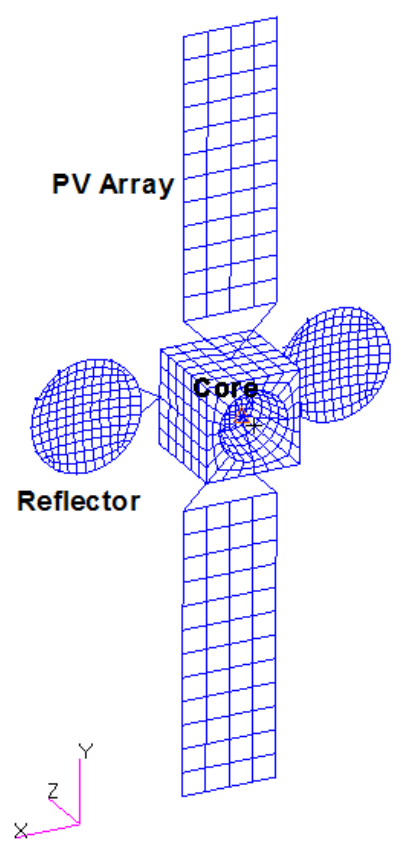

Fig. 1: Generic Satellite with 7,146 degrees of freedom. 

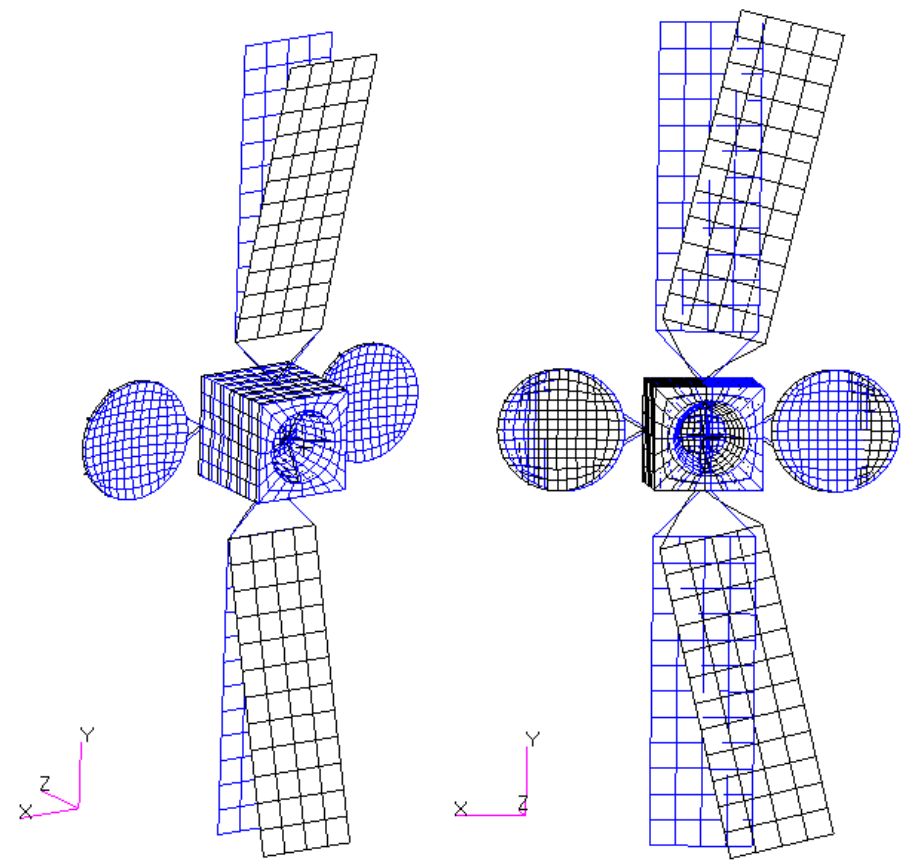

Fig. 2: Elastic Mode 1 (left) and Elastic Mode 2 (right).

Table 1: Generic Satellite Elastic Mode Frequencies

\begin{tabular}{|r|r|c|}
\hline $\begin{array}{c}\text { Flexible Mode } \\
\text { Number }\end{array}$ & $\begin{array}{c}\text { Frequency } \\
\text { (Hz) }\end{array}$ & $\begin{array}{c}\text { Primary } \\
\text { Direction }\end{array}$ \\
\hline 1 & 0.31 & $\mathrm{Z}$ \\
\hline 2 & 0.63 & $\mathrm{X}$ \\
\hline 3 & 0.8 & $\mathrm{Z}$ \\
\hline 4 & 1.79 & $\mathrm{X}$ and $\mathrm{Y}$ \\
\hline 5 & 2.72 & $\mathrm{Z}$ \\
\hline 6 & 2.83 & $\mathrm{Z}$ \\
\hline 7 & 3.49 & $\mathrm{Z}$ \\
\hline 8 & 3.68 & $\mathrm{Z}$ \\
\hline 9 & 3.99 & $\mathrm{Z}$ \\
\hline 10 & 4.24 & $\mathrm{Z}$ \\
\hline 11 & 5.91 & $\mathrm{Y}$ \\
\hline 12 & 6.41 & $\mathrm{Y}$ \\
\hline 13 & 7.46 & $\mathrm{Z}$ \\
\hline 14 & 7.48 & $\mathrm{Z}$ \\
\hline 15 & 9.61 & $\mathrm{Z}$ \\
\hline 16 & 9.71 & $\mathrm{Z}$ \\
\hline 17 & 11.75 & $\mathrm{Z}$ \\
\hline 18 & 11.75 & $\mathrm{Z}$ \\
\hline & & \\
\hline
\end{tabular}

\section{Sensor Placement Algorithms}

Most previous studies comparing various TAM representations have used the same sensor set for each TAM. However, the sensor set has a tremendous influence on the robustness of each TAM. The modal TAM requires that the target modes partitioned to the sensor set are as linearly independent as possible, while the static TAM requires a sensor set that renders the mode shapes linearly independent and captures most of the mass of the structure. Three techniques are used to generate sensor locations in this work: a modified version of modal kinetic energy, effective independence and a new method that seeks to minimize the condition number of the a- 
set modal matrix. The modal kinetic energy method is best suited for the Static and IRS TAM, while the other two are best suited for the Modal TAM. In each case 108 sensors are used for 18 target modes.

\subsection{Modified Modal Kinetic Energy (KE)}

Modal kinetic energy ranks the dynamic importance of each FEM degree of freedom based on a combination of mass and modal displacement. Assuming that the target modes are mass normalized, the fractional contribution of the $i$ th candidate sensor to the $j$ th modal kinetic energy is given by

$$
\left.T_{i j}=\phi_{i j}\left\{M_{i}\right\} \phi_{j}\right\}
$$

where

$\phi_{i j}=$ the $i$ th row of the $j$ th target FEM mode shape

$M_{i}=$ the $i$ th row of the FEM mass matrix

$\phi_{j}=j$ th target mode

The average modal kinetic energy for each DOF is computed by forming the matrix $T$ and averaging each row over all the target modes. In practice, the DOF are ranked, and the most important ones retained such that approximately $90 \%$ of the modal kinetic energy is captured by the sensor set. In this particular case, this criterion resulted in an unreasonably large sensor set. Therefore, the modal kinetic energy calculation was performed one target mode at a time and the top six degrees of freedom were selected for each target mode. Only translational degrees of freedom were considered in this calculation.

\subsection{Effective Independence (Efl)}

The effective independence algorithm aims to place the sensors such that the target modes are as linearly independent as possible, which is required for the Modal TAM. The sensor placement process begins by designating a large set of candidate sensor locations from which the smaller final sensor configuration is selected. There are two approaches that can be taken. In its original form, the effective independence method iteratively reduces the large candidate set down to the desired number of sensor locations [16]. In its most recent form [13], the method starts with a small initial set of sensors and expands it to the desired number. For this work, the original form of the algorithm was used. The candidate sensor set included all translational degrees of freedom. Kammer [16] suggested that optimal sensor placement is achieved when the determinant of the Fisher Information Matrix $Q$ is maximized, where

$$
Q=\phi_{a}^{T} \phi_{a}
$$

and $\phi_{a}$ is the target mode shape matrix partitioned to the sensor degrees of freedom. Maximizing the information matrix determinant will maximize the spatial independence of the target mode partitions. It will also maximize the signal strength of the target modal responses in the sensor output, which is very desirable in the presence of noise. The effective independence of the $i$ th degree of freedom is given by

$$
\begin{aligned}
& E_{D i}=\phi_{a}^{i} Q^{-1} \phi_{a}^{i T} \\
& 0.0 \leq E_{D i} \leq 1.0
\end{aligned}
$$


where $\phi_{a}^{i}$ is the $i$ th row of the target mode partition matrix associated with the $i$ th candidate sensor location. A value of zero indicates that the $i$ th sensor contributes nothing to the linear independence of the target modes or even their observability, and a value of 1.0 indicates that the corresponding sensor is absolutely vital to the independence of the target modes and thus cannot be deleted from the candidate set. The Efl sensor set is used in a Modal TAM.

\subsection{Condition Number Sensor Selection (C\#)}

The modal filtering process upon which the Modal TAM is based depends upon the mode shape matrix for the aset degrees of freedom, $\phi_{a}^{T} \phi_{a}$, being invertible. If $\phi_{a}$ has a large condition number, then sensitivity may be encountered when attempting to reconstruct the response at the omitted degrees of freedom from the response at the a-set degrees of freedom. Mayes suggested that one could minimize the sensitivity of the Modal TAM to errors in the measurements at the a-set degrees of freedom by minimizing the condition number of $\phi_{a}$. He has used this criterion to optimize sensor placement in another work presented concurrently with this [17], as well as in a few other unpublished applications.

Mayes also suggested an algorithm that can be used to obtain a sensor set that minimizes the condition number of $\phi_{a}$. One begins with an initial sensor set, typically a visualization set, of sensors that must be included in the test. Each of the other sensors is then considered one at time (or triax by triax), and the condition number of the mode shape matrix with that sensor included is computed and stored. The process is repeated for all of the candidate sensors, and the sensor that reduces the condition number of the mode shape matrix the most is retained. The process can then be repeated to add another sensor or group of sensors until the desired number of sensors is reached, or until the condition number ceases to decrease significantly. Although this process of locally-optimal steps does not guarantee that a global optimum sensor set is obtained, the method has proven useful [17]. Both this sensor placement strategy, herein denoted the C\#, and the correlation between the condition number of the mode shape matrix and sensitivity to errors in the mode shapes will be evaluated in the following sections. The C\# sensor set is also used in a Modal TAM.

\subsection{Sensor Locations for Satellite Model}

The Static TAM sensor locations, found using the KE method are shown in Fig. 3. Each diamond represents a sensor in the $\mathrm{X}, \mathrm{Y}$, or $\mathrm{Z}$ direction, or a combination of the three. It is important to note that no engineering judgment was used to supplement the modified modal kinetic energy algorithm in an effort to avoid biasing the result. However, it was discovered that the lumped masses had to be instrumented to provide an accurate Static TAM because they represent such a large portion of the mass of the structure. Therefore, these lumped masses were manually included in the KE sensor set used for the static TAM. This sensor set is also used for the IRS TAM.

The Modal TAM Efl and C\# sensor set locations are also shown in Fig. 3. Effective independence favors the edges of the satellite, and does not place any sensors on the satellite core. The C\# sensor placement approach was initiated with a visualization set comprised of 26 sensors on the edges of the PV arrays and reflectors, and primarily selected sensors on the satellite core. 

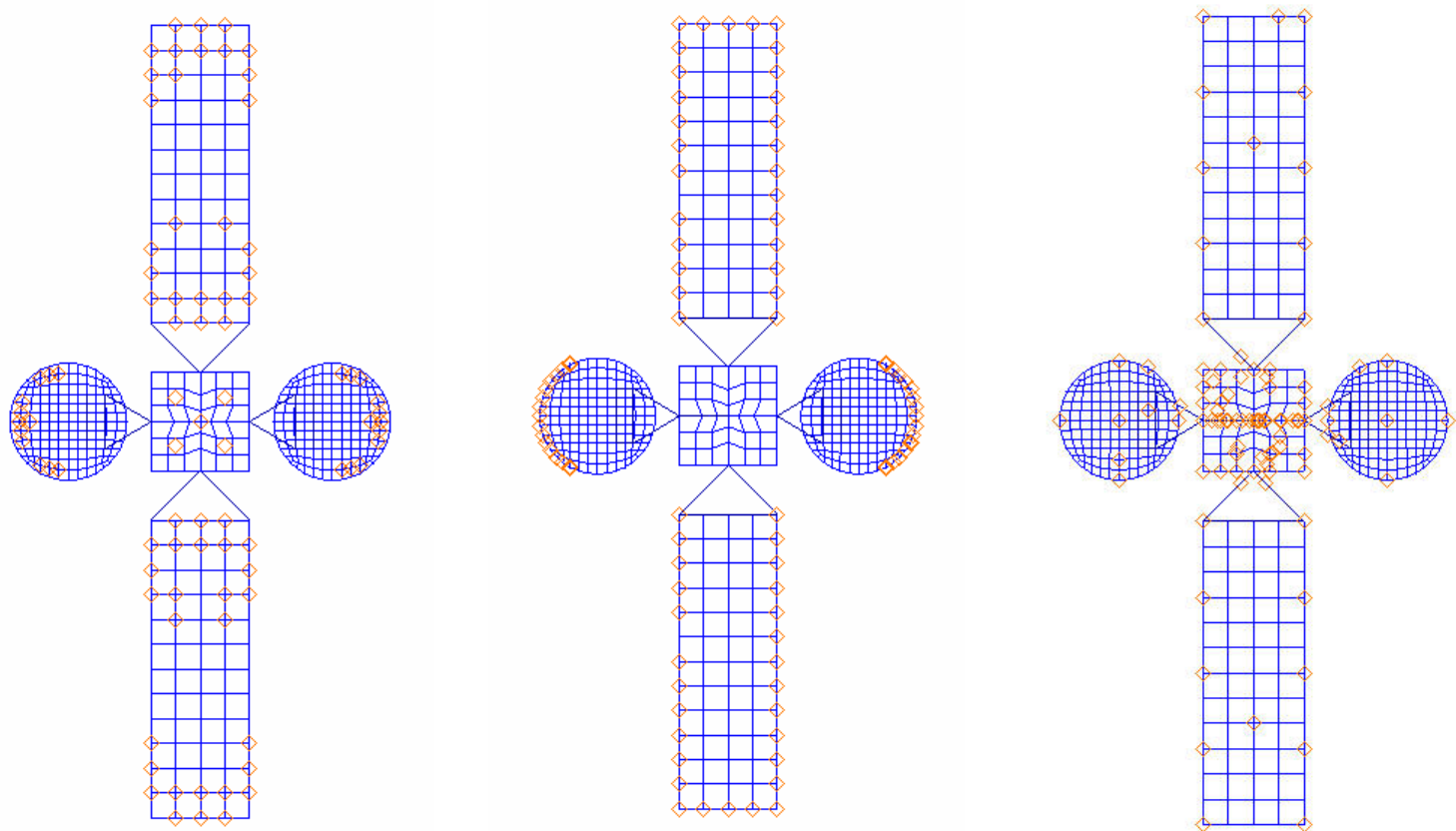

Fig. 3: Static/IRS (left), Modal EFI (middle) and Modal Condition Number TAM sensor locations.

In order to examine the o-set dynamics as a source of TAM sensitivity, as discussed by Gordis [14], the a-set degrees of freedom, or sensor locations, determined using each of the KE and Efl sensor placement techniques were constrained and the modes of the resulting constrained systems were computed. Table 2 lists the natural frequencies of the target modes of the actual system, and the natural frequencies of the system with the a-set DOF constrained corresponding to each sensor set. The Static/IRS TAM sensor set produces natural frequencies approximately $5 \mathrm{~Hz}$ away from the last two system target modes. According to Gordis, this result indicates that the IRS will not be very robust. Both Gordis [14], and Blelloch and Vold [15] have extrapolated this theory to apply to any TAM that approximates the inertia terms, such as the Modal TAM. As seen in Table 2, the Modal Efl TAM a-set produces modes that are interspersed with the system natural frequencies. According to their theory, the Modal TAM for this sensor set should be highly sensitive to errors in the test mode shapes.

Table 2: Constrained A-set Natural Frequencies

\begin{tabular}{|c|c|c|}
\hline $\begin{array}{c}\text { System } \\
\text { Target Mode } \\
\text { Frequencies (Hz) }\end{array}$ & $\begin{array}{c}\text { Static/IRS } \\
\text { Constrained A-set } \\
\text { Frequencies (Hz) }\end{array}$ & $\begin{array}{c}\text { Modal EfI } \\
\text { Constrained A-set } \\
\text { Frequencies (Hz) }\end{array}$ \\
\hline 0.31 & 16.84 & 0.37 \\
\hline 0.63 & 16.84 & 1.18 \\
\hline 0.80 & 17.45 & 1.88 \\
\hline 1.79 & 17.45 & 2.65 \\
\hline 2.72 & 17.76 & 3.19 \\
\hline 2.83 & 17.76 & 5.91 \\
\hline 3.49 & 19.56 & 17.35 \\
\hline 3.68 & 19.56 & 17.35 \\
\hline 3.99 & 23.85 & 17.61 \\
\hline 4.24 & 25.03 & 17.64 \\
\hline 5.91 & 27.14 & 17.76 \\
\hline 6.41 & 27.14 & 17.76 \\
\hline 7.46 & 31.33 & 19.33 \\
\hline 7.48 & 31.33 & 19.35 \\
\hline 9.61 & 31.34 & 21.52 \\
\hline 9.71 & 31.34 & 21.52 \\
\hline 11.75 & 35.03 & 21.92 \\
\hline & &
\end{tabular}




\section{Pre-Test TAM/FEM Correlation Results}

The metrics used to measure TAM/FEM correlation are orthogonality

$$
O=\left[\phi_{F E M}\right]^{T}\left[\tilde{M}_{T A M} \llbracket \phi_{F E M}\right]
$$

cross orthogonality

$$
C O=\left[\phi_{F E M}\right]^{T}\left[\tilde{M}_{T A M}\right]\left[\phi_{T A M}\right]
$$

and a frequency comparison between the TAM and FEM mode shapes

$$
f_{\text {error }}=\frac{f_{F E M}-f_{\text {TAM }}}{f_{F E M}} * 100
$$

where $\phi_{T A M}$ represents the TAM target mode shapes calculated using the reduced mass and stiffness matrices, $\tilde{M}_{T A M}$ represents the reduced mass matrix, and $\phi_{F E M}$ represents the FEM target mode shapes partitioned to the sensor degrees of freedom.

Each sensor set provided acceptable TAM/FEM correlation when the perfect FEM modes were used. Table 3 displays the frequency error for the Static and IRS TAMs. The maximum frequency error for the Static TAM is approximately $2.3 \%$, and is much smaller for the IRS TAM. As expected, the Modal TAMs reproduce the natural frequencies of the 18 target modes perfectly, and result in perfect orthogonality. Fig. 4 displays the orthogonality for the Static and IRS TAMs. Each TAM passes the criteria for TAM/FEM correlation. The maximum off-diagonal orthogonality and cross-orthogonality values for the Static TAM were 0.05 and 0.031 respectively, while the corresponding values for the IRS TAM were all less than 0.01 .

Table 3: Static and IRS TAM frequency error

\begin{tabular}{|c|c|c|c|c|}
\hline $\begin{array}{c}\text { FEM Target } \\
\text { Mode } \\
\text { Number }\end{array}$ & $\begin{array}{c}\text { FEM } \\
\text { Frequency } \\
\mathbf{( H z )}\end{array}$ & $\begin{array}{c}\text { TAM Target } \\
\text { Mode } \\
\text { Number }\end{array}$ & $\begin{array}{c}\text { Static TAM } \\
\text { Frequency Error } \\
\mathbf{( \% )}\end{array}$ & $\begin{array}{c}\text { IRS TAM } \\
\text { Frequency Error } \\
\mathbf{( \% )}\end{array}$ \\
\hline 1 & 0.31 & 1 & 0.00 & 0.00 \\
\hline 2 & 0.63 & 2 & 0.00 & -0.01 \\
\hline 3 & 0.80 & 3 & 0.00 & 0.00 \\
\hline 4 & 1.79 & 4 & 0.00 & 0.00 \\
\hline 5 & 2.72 & 5 & -0.10 & 0.00 \\
\hline 6 & 2.83 & 6 & -0.11 & 0.00 \\
\hline 7 & 3.49 & 7 & -0.15 & 0.00 \\
\hline 8 & 3.68 & 8 & -0.17 & 0.00 \\
\hline 9 & 3.99 & 9 & -0.43 & 0.00 \\
\hline 10 & 4.24 & 10 & -0.37 & 0.00 \\
\hline 11 & 5.91 & 11 & -0.09 & 0.00 \\
\hline 12 & 6.41 & 12 & -0.11 & 0.00 \\
\hline 13 & 7.46 & 13 & -1.50 & 0.00 \\
\hline 14 & 7.48 & 14 & -1.51 & 0.00 \\
\hline 15 & 9.61 & 15 & -2.23 & 0.00 \\
\hline 16 & 9.71 & 16 & -2.31 & 0.00 \\
\hline 17 & 11.75 & 17 & -0.16 & 0.00 \\
\hline 18 & 11.75 & 18 & -0.16 & 0.00 \\
\hline
\end{tabular}



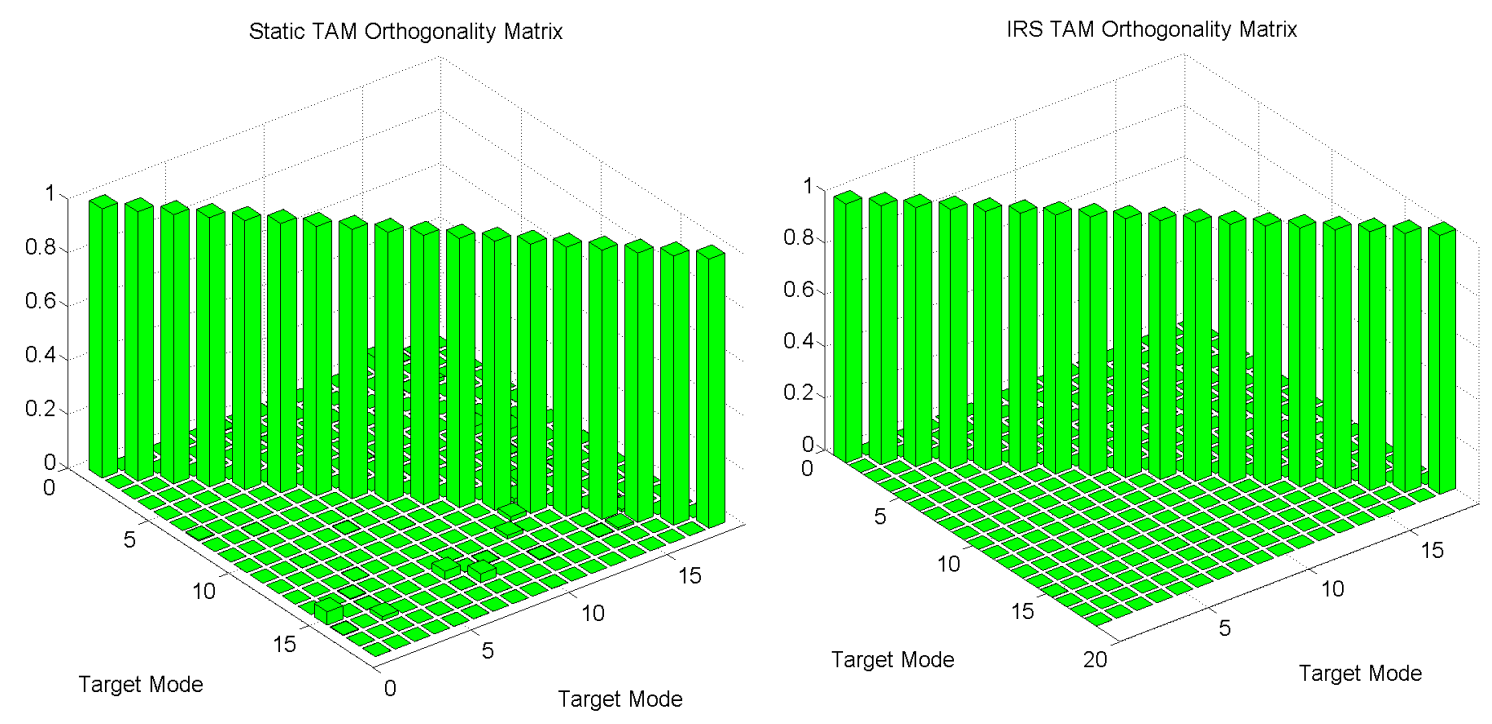

Fig. 4: FEM-TAM Orthogonality Matrices for Static and IRS TAMs.

\section{Probabilistic Study of Test-Analysis Correlation}

\subsection{Noise Model}

In practice, model updating and validation are complicated by the fact that experimental measurements are contaminated with both random noise and systematic errors. Errors are introduced due to electrical noise in the measurement transducers, sensor calibration errors, errors in the positioning and alignment of the sensors and errors, biases and variability in the modal parameter identification routines to name just a few. Generally applicable models for these types of errors are not available, yet there is increasing interest in quantifying measurement uncertainty [18]. For example, a number of recent works have sought to estimate the variance of the modal parameters estimated by state of the art modal parameter estimation routines [19-21].

For the present study, experimentally measured mode shapes were simulated by adding uniformly distributed noise to each finite element mode shape. This model presumes no information about the nature of the errors contaminating the measured mode shapes, except that it is bounded by a certain value. Each mode shape was noise contaminated by adding a vector of uniformly distributed random numbers scaled to $2 \%$ of the maximum value of each mode shape. The same noise-contaminated mode vectors were used to study each TAM.

$$
\begin{gathered}
\{\phi\}_{\text {test }}=\{\phi\}_{F E M}+\{\phi\}_{\text {noise }} \\
\{\phi\}_{\text {noise }}=n^{*} e^{*}\{U\}
\end{gathered}
$$

where

$\{\phi\}_{\text {test }}=$ FEM target mode shape with added noise

$\{\phi\}_{\text {FEM }}=$ FEM target mode shape

$\{\phi\}_{\text {noise }}=$ vector of noise added to FEM target mode shape

$n=$ noise level, in this study $n=0.02$ 
$e=$ scaling factor which is equal to the maximum value of the mode shape $\{\phi\}_{F E M}$

$\{U\}=$ vector of independent uniformly distributed random numbers between -1 and 1 .

The actual noise profile obtained in a real experiment is much more complicated than that represented here, yet one would expect the magnitude of the uncertainty in each element of an experimentally measured mode shape to be significantly greater than $2 \%$. For example, accelerometer calibration factors are typically reported with a $95 \%$ confidence interval of $\pm 4 \%$, so typical uncertainty due to calibration alone is greater than the maximum uncertainty used here.

\subsection{Statistical Results}

The test-orthogonality $(\mathrm{O})$ between the test mode shapes, utilizing the TAM mass matrix

$$
O=\left[\phi_{\text {test }}\right]^{T}\left[\tilde{M}_{\text {TAM }}\right]\left[\phi_{\text {test }}\right]
$$

was computed for each of the TAMs using the simulated (noise-contaminated) test mode shapes. The result found using the Static TAM mass matrix is displayed in Fig 5. Comparing this with the orthogonality matrix computed using perfect mode shapes in Fig. 4, almost all the off-diagonal terms have increased and a few exceed the test-orthogonality criteria. This result represents the orthogonality matrix that might be obtained with a single estimate of the test mode shape matrix. The result obtained in a given test depends on the specific value of the noise obtained in each mode shape coefficient, so one might pass, or fail to pass the test-exit criteria, depending on the particular pattern of errors in the test mode shapes. To obtain a complete picture of this random process, one should compute the probability of passing or failing the orthogonality test given the assumed noise model. This was done by performing a Monte Carlo Simulation (MCS). The test-orthogonality calculation was repeated for 10,000 random noise profiles, and the maximum and mean off-diagonal terms in the orthogonality computation were stored for each trial. Model validation criteria require that the maximum value in the test-orthogonality matrix be less than some threshold for a model to be considered valid, so one can use these values to compute the probability of passing the test-orthogonality metrics in the presence of noise. The mean of the off-diagonal terms gives a view of the average performance of the TAM.

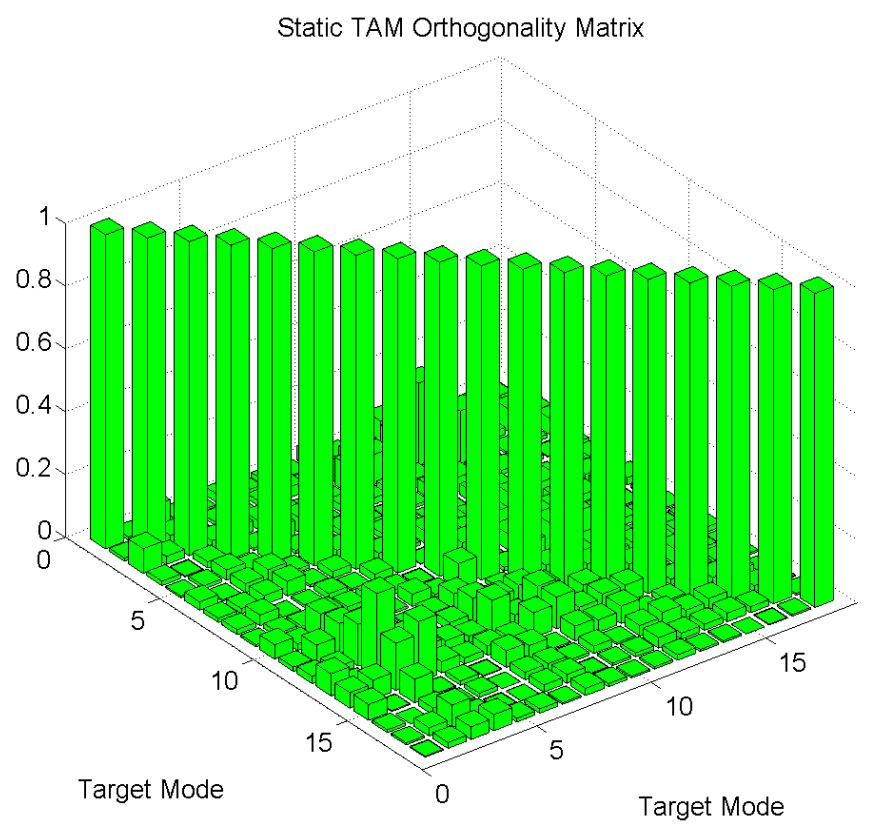

Fig. 5: Static TAM Test-Orthogonality Matrix using simulated test mode shapes. 
Fig. 6 shows a histogram of the maximum off-diagonal terms obtained in 10,000 replications of the MCS. One can observe that the maximum off-diagonal term exceeds the Air Force validation criteria of 0.10 in most of the tests. Test personnel utilizing this TAM would have about a $1-3 \%$ chance of meeting the test exit criteria, depending on the specific errors obtained in the set of measured modes, or in other words, depending on random chance. Similar trends were observed for the cross-orthogonality. Recall that the simulated experimental mode shapes are merely noise contaminated copies of the true mode shapes, so it would be a mistake to update the finite element model to better correlate with these mode shapes.

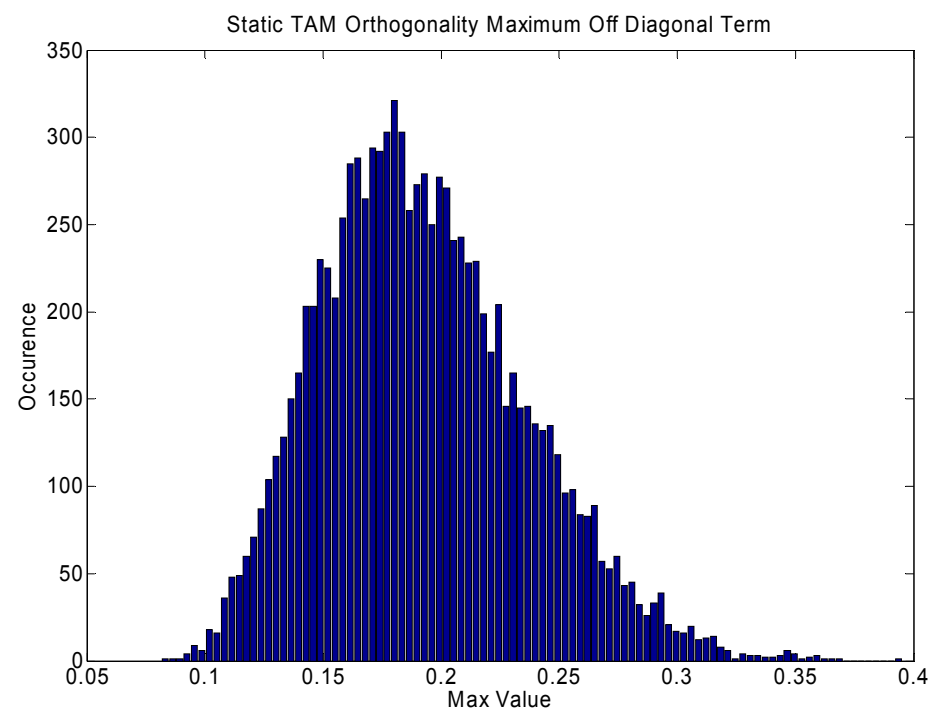

Fig. 6: Histogram of Maximum Off-Diagonal Term in Static TAM Test-Orthogonality.

Probability density functions (PDFs) of the MCS results were estimated using the method in [22]. Fig. 7 shows estimates of the PDFs of the maximum off-diagonal term in the test orthogonality calculation for the Static, IRS and Modal Effective Independence (EFI) and Modal Condition Number (C\#) TAMs. The probability of obtaining a passing maximum off-diagonal value (i.e. less than 0.1 ) is quite small for both the Static and IRS TAMs, so these TAMs would almost always indicate an uncorrelated model because of the noise in the test mode shapes. On the other hand, the PDF indicates that the test mode shapes satisfy the orthogonality criteria most of the time when the Modal TAM is used. The Modal TAM gives similar results whether effective independence or the condition number approach is used for sensor placement. Note however, that the condition number algorithm was quite sensitive to the initial sensor set. Other initial sets gave higher or lower condition numbers and sensitivity than that presented here in Fig. 7.

Figure 8 shows estimated PDFs of the mean of the off-diagonal terms for the Static and Modal TAMs. The PDF of the IRS mean off-diagonal terms was much farther to the right, so it was not included in the figure. Comparison with Fig. 6 reveals that the average off-diagonal term is about an order of magnitude lower than the maximum. This result, and inspection of individual noise-contaminated orthogonality results reveals that a few modes tend to be responsible for the large maximum off-diagonal terms. The most common offender was the coupling between mode 3 and all the other target modes. The MCS data is displayed numerically in Table 4, which lists the mean and standard deviation of the maximum and average off-diagonal terms obtained using each TAM. The standard deviation values give a measure of the variability one can expect in the maximum orthogonality coefficient. The results indicate the Modal TAM is clearly the least sensitive to errors, or the most robust, while the IRS TAM is the most sensitive. The results of two additional sensor sets found using the Condition number algorithm are also presented. These sets were obtained by starting with the 3 sensors possessing the highest effective independence, and the 15 lumped masses on the core of the satellite, and were dubbed " $3 \mathrm{EFl}$ " and "15 LM", respectively. These results show that the performance of the condition number approach depends upon the initial sensor set, yet in all cases, the resulting TAM passed the orthogonality criteria most of the time. It is also interesting to note that the condition number of the mode shape matrix does not perfectly predict the sensitivity of the TAM to errors. For example, the Modal EFI TAM and two of the Modal C\#TAMs had about the same sensitivity to noise, yet their condition numbers ranged from 3.8 to 16 . 
Figure 9 shows a plot of mode 3 contaminated with the error model discussed previously, and then expanded to the full FEA model size using both the Static TAM transformation matrix and the Modal TAM transformation matrix. The perfect mode shape is also shown for comparison. The Static TAM fails to correctly reproduce a reasonable approximation of the reflector motion at the o-set degrees of freedom from the simulated test mode shape, illustrating the sensitivity of the static transformation. The modal TAM gives a reasonable approximation of the o-set motion using the simulated test modes.

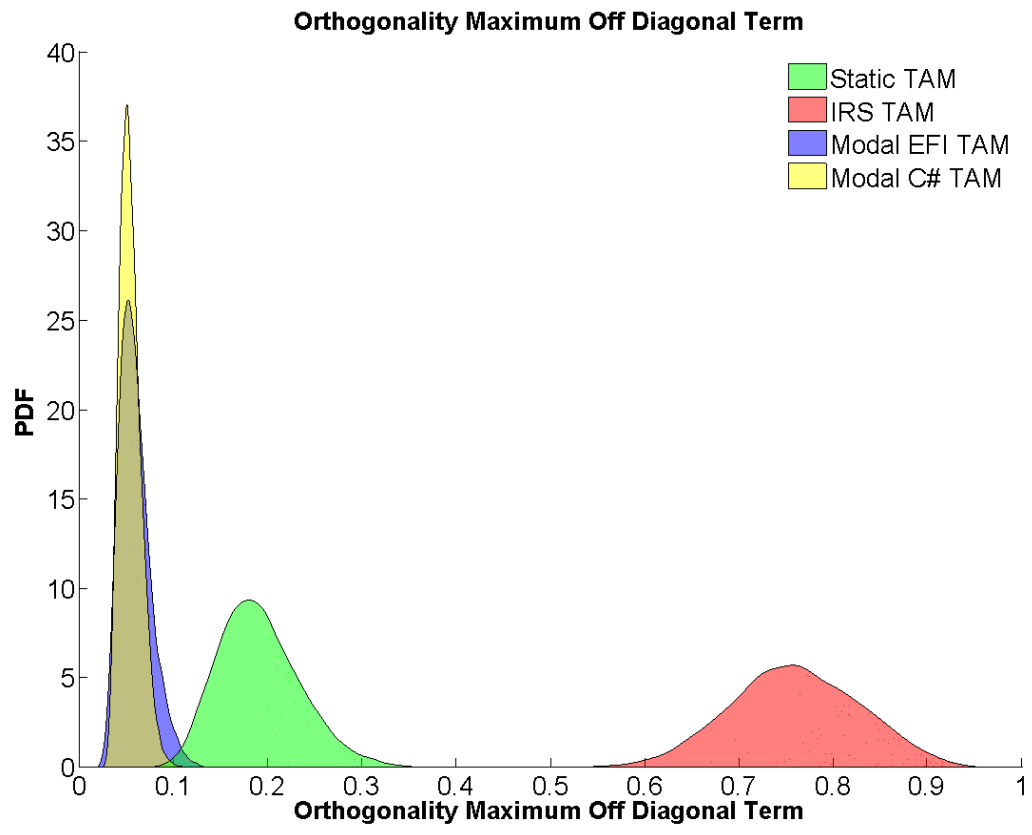

Fig. 7: Estimated Probability Density Functions of Maximum Off-Diagonal term in TestOrthogonality Matrix for Static, IRS, EFI Modal, and Condition Number Modal TAMs.

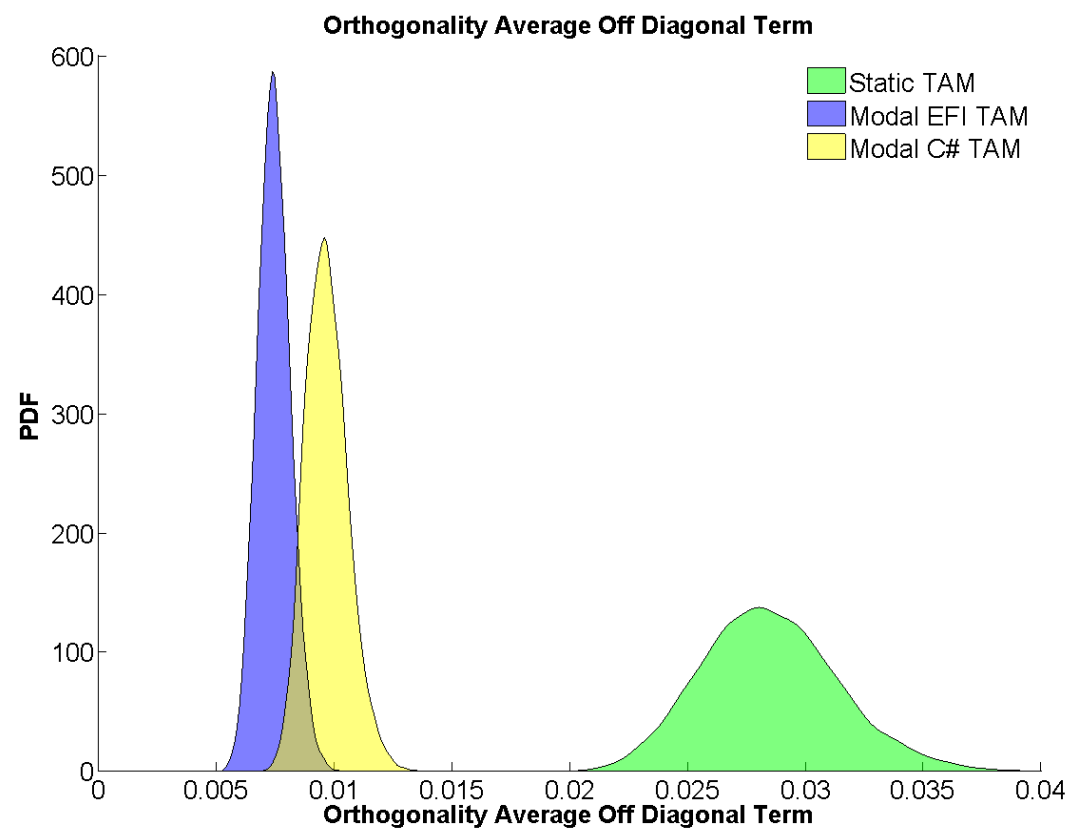

Fig. 8: Estimated Probability Density Functions of Average Off-Diagonal term in TestOrthogonality Matrix for Static, EFI Modal, and Condition Number Modal TAMs. 
Table 4: Static, IRS, and Modal TAM Test-Orthogonality Statistics

\begin{tabular}{|c|c|c|c|c|c|}
\hline & \multicolumn{2}{|c|}{$\begin{array}{c}\text { Maximum } \\
\text { Orthogonality } \\
\text { Off-Diagonal }\end{array}$} & \multicolumn{2}{c|}{$\begin{array}{c}\text { Average } \\
\text { Orthogonality Off- } \\
\text { Diagonal }\end{array}$} & $\begin{array}{c}\text { Condition } \\
\text { Number } \\
\phi_{a}\end{array}$ \\
\cline { 2 - 5 } Mean & STD & Mean & STD & \\
\hline Static & 0.191 & 0.043 & 0.029 & $2.9 \mathrm{E}-03$ & 14.446 \\
\hline IRS & 0.758 & 0.069 & 0.244 & $2.8 \mathrm{E}-02$ & 14.446 \\
\hline Modal EFI & 0.060 & 0.017 & 0.008 & $7.0 \mathrm{E}-04$ & 16.227 \\
\hline $\begin{array}{c}\text { Modal with } \\
\text { Static DOF }\end{array}$ & 0.108 & 0.024 & 0.016 & $1.6 \mathrm{E}-03$ & 14.446 \\
\hline \begin{tabular}{c} 
Modal C\#TAM \\
\hline $\begin{array}{c}\text { Modal C\#TAM } \\
\text { Starting w/3 EFI }\end{array}$
\end{tabular} & 0.055 & 0.011 & 0.010 & $9.0 \mathrm{E}-04$ & 3.759 \\
\hline $\begin{array}{c}\text { Modal C\#TAM } \\
\text { Starting w/15 LM }\end{array}$ & 0.032 & 0.010 & 0.011 & $7.6 \mathrm{E}-04$ & 6.317 \\
\hline
\end{tabular}
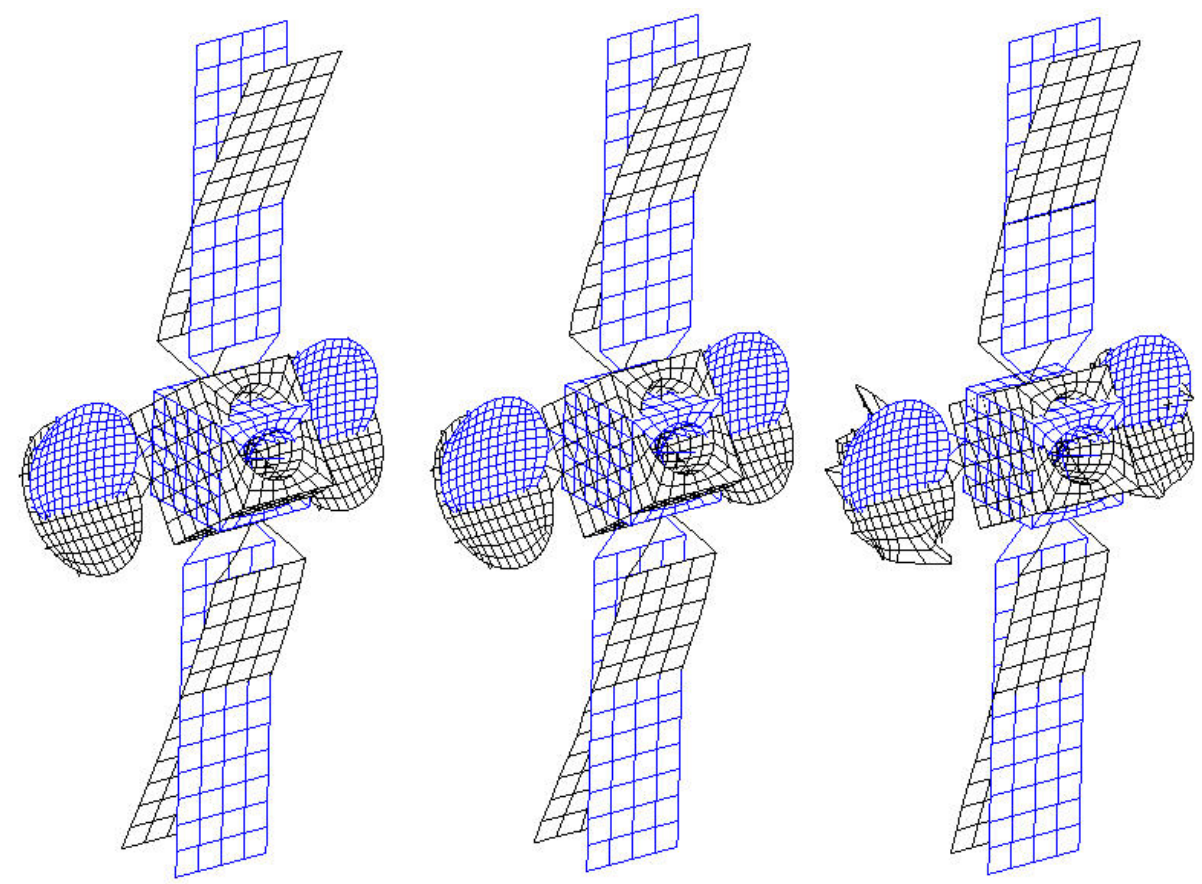

Fig. 9: Perfect Mode Shape 3 (left), Mode Shape with Noise Expanded using Modal EFI TAM (middle), and a Mode Shape with Noise Expanded using Static TAM (right)

As noted previously, the authors are not aware of another work that has compared the Static and Modal TAMs when each had its own optimum sensor set. The importance of the sensor set choice was studied by creating another Modal TAM using the sensor locations that were optimized for the Static TAM. The PDF of the maximum 
off-diagonal term for this sub-optimal Modal TAM is shown in Fig. 10. The corresponding result for the previous Static and Modal EFI TAMs are repeated for comparison. Interestingly, the sensitivity of the Modal TAM increased significantly when it was implemented using the Static TAM sensor locations.

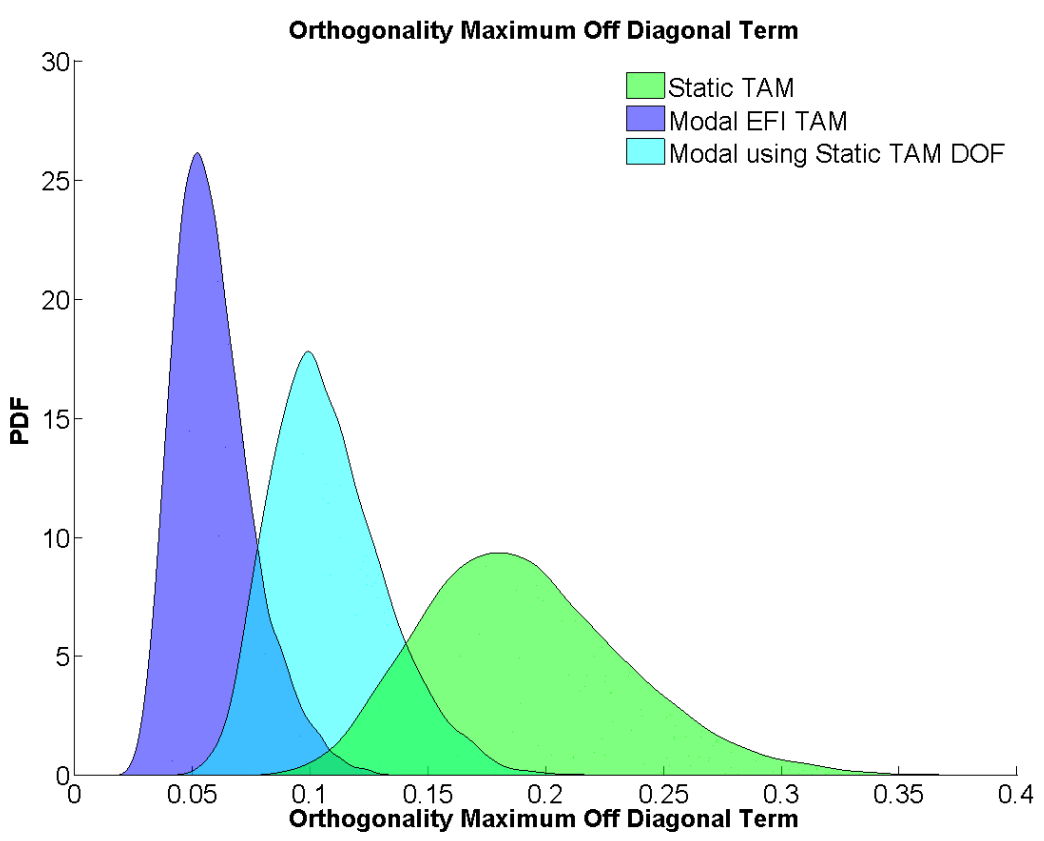

Fig. 10: Estimated Probability Density Functions of Maximum Off-Diagonal Term in TestOrthogonality Matrix for Static, EFI Modal TAM and Modal TAM Created Using the Static TAM Sensor Locations.

\subsection{Discussion}

This study found that the IRS TAM was by far the most sensitive of the TAMs examined, for the assumed error model in the test mode shapes. Its poor performance can perhaps be attributed to the ill- conditioning of the approximated terms in the dynamic reduction equation, as discussed by Gordis [14]. On the other hand, the Modal TAM performed quite well despite the fact that it had natural frequencies that were much closer to the system natural frequencies when its a-set degrees of freedom were constrained (see Table 2).

When evaluating the results presented in Fig. 7 and 8, one should keep in mind that the sensitivity of these TAMs may depend heavily on the problem, and on the noise model for the mode shapes. The authors are not aware of any other works that have examined model correlation using TAMs in a stochastic framework. However, the authors also applied these methods on a real satellite system that had 6 target modes and 120 sensors and obtained similar results. The noise in that case was uniformly distributed with 10 times the amplitude used here, and resulted in the mean of the maximum off-diagonal terms in the cross-orthogonality for the Static and Modal TAMs being 0.23 and 0.11 respectively, so the trends were similar to what was observed here for testorthogonality.

\section{Conclusions}

A review of the literature found a number of papers that have investigated the sensitivity of advanced TestAnalysis Models (TAMs) to errors in the test mode shapes. Their results were summarized, and found to be far from conclusive in identifying a certain TAM as the best in all situations. Nevertheless, urban legend still declares that the advanced TAMs, such as the Modal, Hybrid and IRS TAMs are sensitive to errors in the test mode shapes. One theory [14] as to why this is the case, particularly for the IRS TAM, was reviewed. 
The sensitivity of the Static, IRS, and Modal TAMs was studied in a stochastic framework using a generic satellite model. Eighteen target modes covering a continuous frequency band were validated using 108 sensor locations. Measurement errors were modeled by contaminating the FEM mode shapes with a small level of uniformly distributed random noise, representing the net uncertainty in the mode shapes due to calibration errors, modal parameter estimation errors, etc... A Monte Carlo simulation was performed and statistics were collected for the maximum and average off-diagonal terms in the test-orthogonality calculations. The cross-orthogonality between the test and FEM was not reported, yet it was found to follow similar trends.

The system studied here exhibited considerable sensitivity, so the Static and IRS TAMs were observed to have a small probability of passing the test-orthogonality criteria, even though the test mode shapes were contaminated with a relatively small level of noise. While one might argue that this result could be specific to the problem or the noise model used here, it certainly gives great cause for concern. In any event, these results show that the industry standard orthogonality metric can be sensitive to errors in the test mode shapes in some situations. Failing to consider the effect of these errors when sensitivity is important can reduce the model correlation and updating process to a game of random chance, and the probability of succeeding might be quite low, even if one has a perfect finite element model. For example, the tails of the histogram in Fig. 6 show that random noise can introduce a situation where a Static TAM can pass test-orthogonality with the maximum off-diagonal term below 0.1 , or indicate a strong failure with an off-diagonal term greater than 0.3. Either value could be obtained in a given test depending entirely on random chance! This could cause significant difficulty for experimentalists if they are not apprised of this phenomenon.

From a model correlation perspective, this sensitivity could lead an analyst to correlate a model to noise in the measurements, or to risk not being able to generate a correlated model at all. One might also seek to modify the TAM procedure or sensor selection method to obtain a better result, yet these efforts could only achieve a random perturbation that meets the correlation criteria, and would not benefit the modeling efforts significantly.

Fortunately, the situation is relatively easy to treat stochastically, so one can easily obtain a quantitative estimate of the effect of measurement errors on the validation process and develop remedies or alternate validation strategies. One could also use the methods presented here to define the criteria under which model correlation is possible for a certain TAM and a certain level of sensor noise. In doing so, it will be important to obtain an accurate model for the measurement errors, and this probably will not be a trivial task. Given the potential magnitude of the problem, one is probably better off with an approximate noise model rather than no noise model at all.

This study also addressed a theory [14] regarding TAM sensitivity, and demonstrated a case for which the Modal TAM does not seem to be overly sensitive, even when the constrained a-set natural frequencies are interspersed with the system target mode natural frequencies. However, the theory did seem to hold for the IRS TAM, which was affected dramatically by the measurement errors.

The authors would like to emphasize that the purpose of this study is not to determine definitively which TAM is the most sensitive. The performance of each TAM might be dependent on the model, the number and character of the target modes selected, and the sensor locations. In this example, the success of the Static TAM depended on the ability to instrument the lumped masses. While every effort was made to use a sensor placement technique that accommodated this, another technique may have yielded better results. The purpose of this paper is rather to deliver an approach to study sensitivity and TAM performance from a probabilistic viewpoint, and it is hoped that this will allow engineers to make more informed decisions regarding TAM sensitivity.

\section{REFERENCES}

1. Hasselman, T.K., R.N. Coppolino, and D.C. Zimmerman. Criteria for Modeling Accuracy: A State-of-thePractice Survey. in 18th International Modal Analysis Conference. 2000. San Antonio, TX.

2. NASA, NASA-STD-5002, Loads Analyses of Spacecraft and Payloads, in NASA-STD-5002. 1996, NASA.

3. $\quad$ Guyan, R.J., Reduction of Mass and Stiffness Matrices. AIAA Journal, 1965. 3(2): p. 380.

4. Kammer, D.C., B.M. Jensen, and D.R. Mason, Test-Analysis Correlation of the Space Shuttle Solid Rocket Motor Center Segment. Journal of Spacecraft and Rockets, 1989. 26(4): p. 266-273.

5. Kammer, D.C., Test-Analysis Model Development Using an Exact Modal Reduction. International Journal of Analytical and Experimental Modal Analysis, 1987. 2(4): p. 174-179. 
6. Kammer, D.C., A Hybrid Approach to Test-Analysis-Model Development for Large Space Structures. Journal of Vibration and Acoustics, 1991. 113(3): p. 325-332.

7. O'Callahan, J. A Procedure for an Improved Reduced System (IRS) Model. in 7th International Modal Analysis Conference. 1989. Las Vegas, NV: Union College.

8. O'Callahan, J., P. Avitable, and R. Riemer. System Equivalent Reduction Expansion Process. in 7th International Modal Analysis Conference. 1989. Las Vegas, NV.

9. Chung, Y.T. and S. Simonian. Assessments of Model Correlation Using Dynamic Reduction and Static Reduction. in Aerospace Technology Conference. 1988: SAE Technical Paper 881527.

10. Freed, A.M. and C.C. Flanigan. A Comparison of Test-Analysis Model Reduction Methods. in 8th International Modal Analysis Conference. 1990. Kissimmee, FL.

11. Avitable, P., F. Pechinsky, and J. O'Callahan. Study of Modal Vector Correlation Using Various Techniques for Model Reduction. in 10th International Modal Analysis Conference. 1992. San Diego, CA.

12. Carne, T.G. and C.R. Dohrmann. A Modal Test Design Strategy for Model Correlation. in 13th International Modal Analysis Conference. 1995. Nashville, TN.

13. Kammer, D.C., Sensor Set Expansion for Modal Vibration Testing. Mechanical Systems and Signal Processing, 2005. 19: p. 700-713.

14. Gordis, J.H. An Analysis of the Improved Reduced System (IRS) Reduction Procedure. in 10th International Modal Analysis Conference. 1992. San Diego, CA.

15. Blelloch, P. and $\mathrm{H}$. Vold. Orthogonality and large models - what's the problem? . in 23rd International Modal Analysis Conference (IMAC XXIII). 2005. Orlando, Florida.

16. Kammer, D.C., Sensor Placement for On-Orbit Modal Identification and Correlation of Large Space Structures. Journal of Guidance Control and Dynamics, 1991. 14(2): p. 251-259.

17. Mayes, R.L., et al. Combining Experimental and Analytical Substructures with Multiple Connections. in 26th International Modal Analysis Conference (IMAC XXVI). 2008. Orlando, Florida.

18. Carne, T.G., D. Todd Griffith, and M.E. Casias, Support conditions for experimental modal analysis. Sound and Vibration, 2007. 41(6): p. 10-16.

19. Guillaume, P., P. Verboven, and S. Vanlanduit. Frequency-Domain Maximum Likelihood Identification of Modal Parameters with Confidence Intervals. in International Seminar on Modal Analysis (ISMA-23). 1998. Leuven, Belgium.

20. Verboven, P., et al. Autonomous Modal Parameter Estimation Based on a Statistical Frequency Domain Maximum Llkelihood Approach. in 19th International Modal Analysis Conference (IMAC-19). 2001. Kissimmee, Florida.

21. Vanlanduit, S., et al., An automatic frequency domain modal parameter estimation algorithm. Journal of Sound and Vibration, 2003. 265: p. 647-661.

22. Silverman, B.W., Density Estimation for Statistics and Data Analysis. Monographs on Statistics and Applied Probability. 1986, London: Chapman and Hall. 